\title{
Intraductal Breast Papillomatosis
}

National Cancer Institute

\section{Source}

National Cancer Institute. Intraductal Breast Papillomatosis. NCI Thesaurus. Code C5201.

A benign breast neoplasm characterized by the proliferation of multiple papillomas within the lumen of the ducts. 